\title{
Viewpoint Ranking Method Based on PageRank in Group Discussion
}

\author{
Jian ZHOU ${ }^{1,2}$, Ning DU ${ }^{1,2,{ }^{*}}$, Li-juan SUN ${ }^{1,2}$ and Fu XIAO ${ }^{1,2}$ \\ ${ }^{1}$ School of Computer, Nanjing University of Posts and Telecommunications, Nanjing, \\ 210023, China \\ ${ }^{2}$ Jiangsu High Technology Research Key Laboratory for Wireless Sensor Networks, \\ Nanjing, 210003, China
}

\begin{abstract}
Keywords: Viewpoint Rank, PageRank, Group Discussion, Hall for Workshop of Meta-synthetic Engineering
\end{abstract}

\begin{abstract}
Real-time display viewpoint ranking information in the divergent discussion process, has a great significance in group discussion of Hall for Workshop of Meta-synthetic Engineering. Since divergent discussion has the characteristics of continuity and randomized complexity, a viewpoint ranking method based on PageRank in group discussion is proposed. On the basis of information organization model, timing factor and semantic relations of experts' comments are taken into account to compute the support degrees among experts. The $P R$ values of experts' viewpoints can be calculated based on the support degrees, and then ranking experts' viewpoints according to the $P R$ values. The example is given to validate the validity and practicability of this method.
\end{abstract}

\section{Introduction}

Hall for Workshop of Meta-synthetic Engineering (HWME) is guided by the meta-synthetic method, it is a decision support system for the purpose of studying the "open complex giant system" and solving complex problems [1-4]. Expert group is a core part of HWME [5], and experts play a major role in presenting, discussing, constructing questions and making final decision. The computer technology and expert knowledge, wisdom, data and various kinds of information are combined by group discussion. Therefore, how to support group discussion will be a key subject to study the development of HWME [6].

Divergent and convergent discussions are included in effective group discussion. At present, in the face of convergent discussion, the consensus algorithm of group decision is proposed in the literature [7], which makes the viewpoints of expert group finally reach the agreement; In the literature [8], based on the complete consistent linguistic judgment matrix, a ranking method is proposed; In the literature [9], a group decision method based on interval two tuple linguistic information is proposed to solve the problem of ranking schemes; In the literature [10], a method for ranking the weights of the schemes based on the interval number complementary judgment matrix is proposed. The domestic and foreign researchers have been fully studied the ranking problem in convergent discussion. In the face of divergent discussion, foreign researchers propose Information system model based on problem [11] and Carneades dispute framework [12]; There have been some research in domestic, e.g. Electronic Common Brain Audiovisual Room (ECBAR) is proposed in the literature [13]; Group discussion environment is proposed in the literature [14]; Cyberspace for Workshop of Meta-synthetic Engineering (CWME) is proposed in the literature [15]. The information organization, process control and the result visualization in the divergent 
discussion have been studied by the domestic and foreign researchers. However, how to rank the experts' viewpoints during the group discussion has not yet been studied.

If the ranking information can be real-time displayed, it would facilitate the analysis to improve the efficiency in divergent discussion. However, on one hand, the continuity of discussion is one of the characteristics of the divergent discussion. Expert group can stimulate thinking in the discussion process, and the continuity of thinking leads to the continuity of discussion, so as far as possible to avoid experts from discussion into voting or expression of preference procedures; On the other hand, the divergent discussion has the characteristic of randomized complexity. With the deepening of discussion, experts' viewpoints vary in the discussion. Meanwhile, the relationship between new comments and other comments cannot be predicted [16]. The above characteristics of divergent discussion bring some difficulties to rank viewpoints. PageRank is a web page ranking algorithm. It can give a global rank based on the importance of the web page. Viewpoint ranking method based on PageRank in group discussion is proposed in this paper. By calculating the PageRank value to rank experts' viewpoints, so our method can improve the efficiency of group discussion.

\section{PageRank Overview}

PageRank [17] is first presented by Sergey Brin and Larry Page, and as the core algorithm of Google. The algorithm references citation analysis methods in the theory of information retrieval, and improves the quality of Google search. It has been recognized by the majority of users.

PageRank is used to evaluate and rank the importance of the web page. It depends on the quality and quantity of the linked web page. In general, if one web page is linked to this web page, the web page is the equivalent of voting for this web page. Web page has more links, the web page gets more votes. If a web page is linked by a higher quality and quantity of page, the higher the PageRank value, then this page is more important.

PageRank is calculated as follows equation (1):

$$
P R\left(W_{j}\right)=(1-d)+d \sum \frac{P R\left(W_{i}\right)}{S\left(W_{i}\right)} .
$$

$P R\left(W_{j}\right)$ is the PageRank value of page $W_{j}$, page $W_{i}$ links to page $W_{j}, S\left(W_{i}\right)$ is the number of page $W_{i}$ linking out, $d$ is the attenuation factor.

\section{Information Organization Model for Divergent Discussion}

Obtaining and analyzing efficiently various data generated in the discussion, it needs the support of information organization model. The information organization model contains that defining, data structures of information and establishing their relations $[18,19]$. In this paper, each comment among experts contains ID, forward ID, spokesman, time, semantic relation, discussion content and other information. The semantic relations in this paper are divided into five types: intensely support (IS), support (S), neutrality $(\mathrm{N})$, opposition $(\mathrm{O})$, intensely opposition (IO).

Experts as the nodes, comments are directed arcs that the connection of different nodes, which constructs digraph $F=(H, L)$, as shown in Figure $1 . H=\left\{h_{1}, h_{2}, \ldots, h_{n}\right\}$ is a collection of experts, $n$ is the total number of experts in the discussion; $L$ represents directed arcs that connect to different nodes. $L=\left\{l_{i j} \mid i, j \in[1, n], i \neq j\right\}$ represents the 
collection of directed edges in a digraph, $l_{i j}$ is the directed edge $h_{i} \rightarrow h_{j}$, representing that expert $h_{i}$ supports the degree of expert $h_{j}$. In the process of discussion, the support degrees among experts will be changed with the advanced of time. On the directed arc, discussion information is represented by triple $(T, A, C)$, where $T_{i j}=(1,2,3, \ldots t)$ is the timing sequence, $A_{i j}=\left(a_{1}, a_{2}, a_{3}, \ldots, a_{t}\right)$ is a collection of semantic relations that expert $h_{i}$ speaks to expert $h_{j}$ (ranking by timing), $C_{i j}=\left(c_{1}, c_{2}, c_{3}, \ldots, c_{t}\right)$ is a collection of the discussion contents that expert $h_{i}$ speaks to expert $h_{j}$ (ranking by timing), where $t$ is the total number that expert $h_{i}$ speaks to expert $h_{j}$. In the discussion, some experts do not express any viewpoints; therefore their viewpoints will not be evaluated by other experts, that is to say experts are not been linked, such as node $h_{4}$ in Figure. 1.

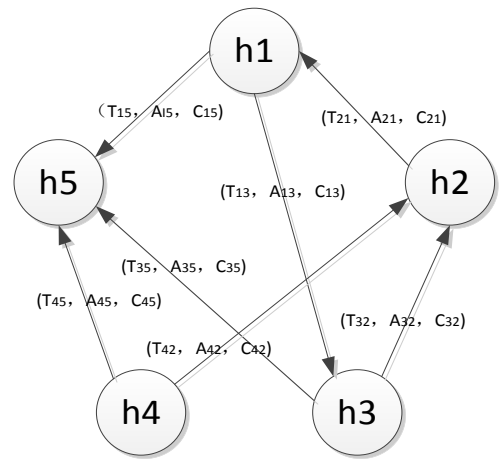

Figure. 1. The digraph of discussion information

The purpose of this paper is to rank experts' viewpoints in group discussion, according to the characteristics of group discussion, based on information organization model, designing the computational methods of support degrees and PageRank values to solve the rank of experts' viewpoints in group discussion.

\section{Rank Viewpoint Based on PageRank}

The ranking method based on PageRank is to calculate the support degrees of experts' viewpoints according to the information among experts. Specifically, when expert $h_{i}$ speaks to expert $h_{j}$, it is equal to express the support degree. The number of support and the support degree are larger, the higher the PageRank value, the more important the expert viewpoint.

First, this section describes how to calculate the support degrees according to the information among experts, and then introduces how to calculate the PageRank values of experts' viewpoints.

\section{Support Degree of Expert Viewpoint}

The directed arc includes some important information: discussion timing, semantic relations and discussion contents, these are represented by triple $(T, A, C)$. In this paper, the support degree is calculated by the weighted arithmetic average operator, which it takes timing factor as weight to make the utility value of semantic relation weighted. The support degree is calculated by the following equation (2): 


$$
e_{i j}=\sum_{k=1}^{t} Q_{k} U\left(a_{k}\right) .
$$

Among them, $t$ is times which expert $h_{i}$ makes comments on expert $h_{j}, a_{k}$ is the $k_{t h}$ semantic relation, $U\left(a_{k}\right)$ is the utility value of semantic relation, as shown in Table 1.

Table 1 . the utility value of semantic relation

\begin{tabular}{|c|c|}
\hline$a$ & $U(a)$ \\
\hline intensely support & 1 \\
\hline support & 0.5 \\
\hline neutrality & 0 \\
\hline opposition & -0.5 \\
\hline intensely opposition & -1 \\
\hline
\end{tabular}

$Q_{k}$ is timing factor, $Q_{k}=\frac{k}{q}$. The greater timing factor closes to the current moment, the greater impact on the current support degrees. Among them, $k$ is times, $q=\frac{t(1+t)}{2}$ is the sum of timing sequences in discussion, $\sum_{k=1}^{t} Q_{k}=1$. Figure 1, for example: expert $h_{3}$ is voted 5 times by expert $h_{1}$, semantic relation collection is $A_{13}=\left\{\mathrm{a}_{1}=\mathrm{O}, \mathrm{a}_{2}=\mathrm{N}, \mathrm{a}_{3}=\mathrm{N}, \mathrm{a}_{4}=\mathrm{N}, \mathrm{a}_{5}=\mathrm{S}\right\}$, according to equation (2), the support degree of expert $h_{3}$ is calculated: $e_{13}=(-0.5) \times \frac{1}{15}+0 \times \frac{2}{15}+0 \times \frac{3}{15}+0 \times \frac{4}{15}+0.5 \times \frac{5}{15}=\frac{2}{15}$.

\section{PR Value of Expert Viewpoint}

The PageRank value is called $P R$ value for short. Google adjusts the rank of web page on the website, according to $P R$ value to improve the quality of search. The $P R$ value represents the importance of expert viewpoint, the higher the $P R$ value, the more important expert viewpoint. This paper ranks viewpoints according to $P R$ value in group discussion.

The $P R$ value of the expert viewpoint is calculated as follows equation (3):

$$
P R\left(h_{j}\right)=(1-d)+d \sum_{h_{i} \in I M P\left(h_{j}\right)} \frac{P R\left(h_{i}\right) e_{i j} w_{i}}{S\left(h_{i}\right)} .
$$

$d$ is attenuation factor; $w_{i}$ is the weight of expert $h_{i}$, that is given according to quality, experience, ability of experts, knowledge structure, and other factors; $\operatorname{IMP}\left(h_{j}\right)=\left\{h_{k} \mid h_{k} \rightarrow h_{j}\right\} \quad$ represents the direct former collection of expert $h_{i}$ in digraph, it is a collection that other experts make some comments on expert $h_{j}$ in this paper; $S\left(h_{i}\right)=\operatorname{card}\left(\operatorname{IMS}\left(h_{i}\right)\right)$ represents the number of experts in a collection $\operatorname{IMS}\left(h_{i}\right)$, $\operatorname{IMS}\left(h_{i}\right)=\left\{h_{k} \mid h_{i} \rightarrow h_{k}\right\}$ represents the direct later collection of expert $h_{i}$ in the digraph, it is a collection of experts who expert $h_{i}$ make a comment on; $P R\left(h_{i}\right)$ is the $P R$ value of expert $h_{i}$. Normally, the original $P R$ values of all experts are $1, d$ is 0.85 [20]. Figure 1, assuming that $P R\left(h_{1}\right)=1, w_{1}=0.2$, the $P R$ value of expert $h_{3}$ is calculated by equation (3): $P R\left(h_{3}\right)=(1-0.85)+0.85 *\left(\frac{1 * \frac{2}{15} * 0.2}{2}\right)=0.16133$. 


\section{Case Analysis}

Taking water pollution prevention of HWME as application background, the expert group discussion visualization system is developed with MyEclipse + MySQL + JSP, as shown in Figure. 2. Two examples are given to validate the validity and practicability of this method.

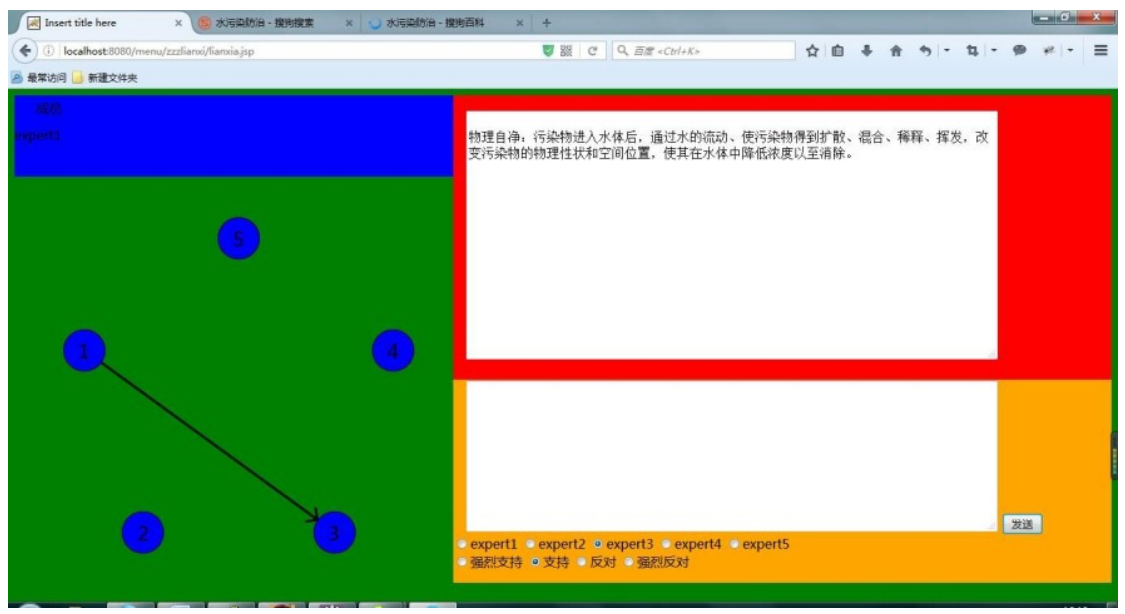

Figure. 2. Group discussion visualization system

Many experts come to HWME to discuss about Taihu water pollution prevention problem. In this paper, viewpoint ranking method based on PageRank is used to rank experts' viewpoints in different group discussions. The utility values of semantic relations are shown in Table 1 , the original $P R$ values are $1, d$ is 0.85 .

(1) In the first discussion, five experts $H=\left\{h_{1}, h_{2}, h_{3}, h_{4}, h_{5}\right\}$ are in HWME, experts' weights are $W=\left(w_{1}, w_{2}, w_{3}, w_{4}, w_{5}\right)=\{0.2,0.1,0.2,0.1,0.4\}$, The digraph based on workshop is shown in Figure. 1, The semantic relations among experts are:

$A_{13}=\left\{a_{1}=O, a_{2}=N, a_{3}=N, a_{4}=N, a_{5}=S\right\}, A_{15}=\left\{a_{1}=O, a_{2}=N, a_{3}=S, a_{4}=S\right\}$,

$A_{21}=\left\{a_{1}=N, a_{2}=N, a_{3}=S\right\}, A_{32}=\left\{a_{1}=I O, a_{2}=N, a_{3}=S, a_{4}=S\right\}, A_{35}=\left\{a_{1}=O, a_{2}=N, a_{3}=N, a_{4}=S\right\}$, $A_{42}=\left\{a_{1}=O, a_{2}=O, a_{3}=N, a_{4}=S\right\}, A_{45}=\left\{a_{1}=O, a_{2}=O, a_{3}=N, a_{4}=S, a_{5}=I S\right\}$.

The support degrees of experts are calculated by equation (2):

$$
\begin{aligned}
& e_{13}=(-0.5) \times \frac{1}{15}+0 \times \frac{2}{15}+0 \times \frac{3}{15}+0 \times \frac{4}{15}+0.5 \times \frac{5}{15}=\frac{2}{15}, e_{15}=(-0.5) \times \frac{1}{10}+0 \times \frac{2}{10}+0.5 \times \frac{3}{10}+0.5 \times \frac{4}{10}=\frac{3}{10} \\
& e_{21}=0 \times \frac{1}{6}+0 \times \frac{2}{6}+0.5 \times \frac{3}{6}=\frac{1}{4}, e_{32}=(-1) \times \frac{1}{10}+0 \times \frac{2}{10}+0.5 \times \frac{3}{10}+0.5 \times \frac{4}{10}=\frac{1}{4}, \\
& e_{35}=(-0.5) \times \frac{1}{10}+0 \times \frac{2}{10}+0 \times \frac{3}{10}+0.5 \times \frac{4}{10}=\frac{3}{20}, e_{42}=(-0.5) \times \frac{1}{10}+(-0.5) \times \frac{2}{10}+0 \times \frac{3}{10}+0.5 \times \frac{4}{10}=\frac{1}{20}, \\
& e_{45}=(-0.5) \times \frac{1}{15}+(-0.5) \times \frac{2}{15}+0 \times \frac{3}{15}+0.5 \times \frac{4}{15}+1 \times \frac{5}{15}=\frac{11}{30} .
\end{aligned}
$$

The $P R$ values of experts' viewpoints are calculated by equation (3):

$$
\begin{aligned}
& P R\left(h_{1}\right)=(1-0.85)+0.85 *\left(\frac{1 * \frac{1}{4} * 0.2}{1}\right)=0.17125, \\
& P R\left(h_{2}\right)=(1-0.85)+0.85 *\left(\frac{1 * \frac{1}{4} * 0.2}{2}+\frac{1 * \frac{1}{20} * 0.1}{2}\right)=0.17338, \\
& P R\left(h_{3}\right)=(1-0.85)+0.85 * \frac{1 * \frac{2}{15} * 0.2}{2}=0.16133, \\
& P R\left(h_{4}\right)=(1-0.85)=0.15000,
\end{aligned}
$$




$$
P R\left(h_{5}\right)=(1-0.85)+0.85 *\left(\frac{1 * \frac{3}{10} * 0.2}{2}+\frac{1 * \frac{3}{20} * 0.2}{2}+\frac{1 * \frac{11}{30} * 0.1}{2}\right)=0.20383 .
$$

The $P R$ values are stable after several iterations, the final $P R$ values is shown in Table 2.

Table 2. The final $P R$ values of experts

\begin{tabular}{|c|c|}
\hline Expert & $P R$ \\
\hline$h_{1}$ & 0.15326 \\
\hline$h_{2}$ & 0.15354 \\
\hline$h_{3}$ & 0.15174 \\
\hline$h_{4}$ & 0.15000 \\
\hline$h_{5}$ & 0.15818 \\
\hline
\end{tabular}

From Table 2, we can rank the current experts' viewpoints: $h_{5}>h_{2}>h_{1}>h_{3}>h_{4}$. From Figure 1, we can see that expert $h_{1}$, expert $h_{3}$ and expert $h_{4}$ make a comment on expert $h_{5}$. Expert $h_{5}$ is supported by three experts in general. Expert $h_{3}$ and expert $h_{4}$ also make a comment on expert $h_{2}$. Similarly, expert $h_{2}$ is supported by two experts in general. Therefore, the $P R$ value of expert $h_{5}$ is greater than the $P R$ value of expert $h_{2}$. Expert $h_{2}$ only makes a comment on expert $h_{1}$, expert $h_{2}$ has attitudes to expert $h_{1}$ from neutral to support. Expert $h_{1}$ has attitudes to expert $h_{3}$ from oppose to support, so the support degree of expert $h_{1}$ is greater than expert $h_{3}$ and the $P R$ value is so. Because expert $h_{4}$ does not express viewpoints, so the $P R$ value is minimum.

(2) In the Second discussion, more experts participate in the discussion. there are ten experts $H=\left\{h_{1}, h_{2}, h_{3}, h_{4}, h_{5}, h_{6}, h_{7}, h_{8}, h_{9}, h_{10}\right\}$ participating in the discussion, their weights are $W=\left(w_{1}, w_{2}, w_{3}, w_{4}, w_{5}, w_{6}, w_{7}, w_{8}, w_{9}, w_{10}\right)=\{0.15,0.1,0.05,0.15,0.1,0.05,0.1,0.15,0.05,0.1\}$, the digraph is shown in Figure. 3, the semantic relations among experts as follows:

$$
\begin{aligned}
& A_{12}=\left\{a_{1}=O, a_{2}=N, a_{3}=N, a_{4}=N, a_{5}=S\right\}, A_{15}=\left\{a_{1}=I O, a_{2}=O, a_{3}=O\right\}, \\
& A_{17}=\left\{a_{1}=O, a_{2}=N, a_{3}=S, a_{4}=S\right\}, A_{21}=\left\{a_{1}=I O, a_{2}=O, a_{3}=N\right\}, \\
& A_{24}=\left\{a_{1}=N, a_{2}=N, a_{3}=S, a_{4}=S, a_{5}=S\right\}, A_{25}=\left\{a_{1}=O, a_{2}=S, a_{3}=I S\right\}, \\
& A_{29}=\left\{a_{1}=O, a_{2}=N, a_{3}=S\right\}, A_{31}=\left\{a_{1}=O, a_{2}=N, a_{3}=S, a_{4}=S\right\}, \\
& A_{34}=\left\{a_{1}=O, a_{2}=N, a_{3}=S\right\}, A_{37}=\left\{a_{1}=I O, a_{2}=O, a_{3}=N\right\}, \\
& A_{38}=\left\{a_{1}=O, a_{2}=O, a_{3}=N, a_{4}=N\right\}, A_{45}=\left\{a_{1}=I O, a_{2}=N, a_{3}=N, a_{4}=S, a_{5}=S\right\}, \\
& A_{58}=\left\{a_{1}=O, a_{2}=O, a_{3}=N, a_{4}=N, a_{5}=S\right\}, A_{62}=\left\{a_{1}=I O, a_{2}=I O, a_{3}=I O, a_{4}=N, a_{5}=N\right\}, \\
& A_{64}=\left\{a_{1}=I O, a_{2}=I O, a_{3}=N, a_{4}=N\right\}, A_{78}=\left\{a_{1}=I O, a_{2}=O, a_{3}=O, a_{4}=N, a_{5}=N\right\}, \\
& A_{74}=\left\{a_{1}=O, a_{2}=O, a_{3}=N, a_{4}=N\right\}, A_{81}=\left\{a_{1}=O, a_{2}=N, a_{3}=S\right\}, \\
& A_{92}=\left\{a_{1}=N, a_{2}=N, a_{3}=S\right\}, A_{93}=\left\{a_{1}=O, a_{2}=N, a_{3}=N, a_{4}=S\right\}, \\
& A_{910}=\left\{a_{1}=I O, a_{2}=O, a_{3}=N, a_{4}=N\right\}, A_{101}=\left\{a_{1}=N, a_{2}=N, a_{3}=S, a_{4}=S, a_{5}=I S\right\}, \\
& A_{103}=\left\{a_{1}=O, a_{2}=O, a_{3}=N, a_{4}=N\right\}, A_{109}=\left\{a_{1}=O, a_{2}=O, a_{3}=N\right\} .
\end{aligned}
$$




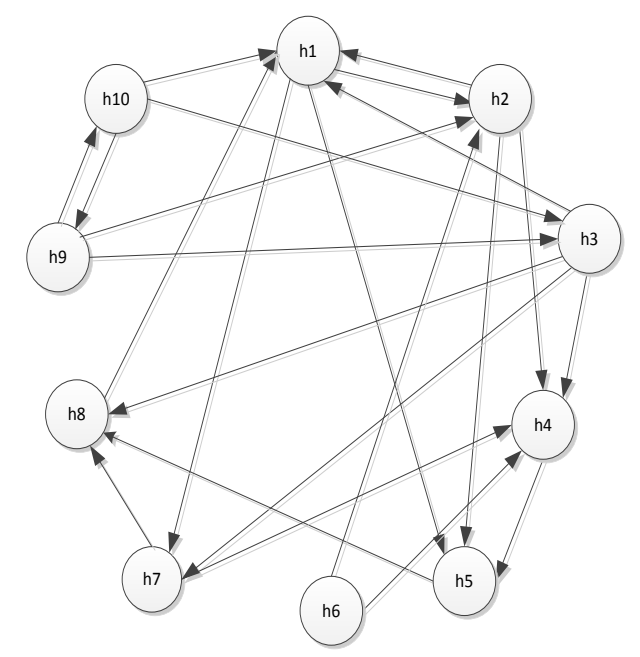

Figure. 3. The digraph of discussion information in many experts

According to equation (2), the support degrees among the experts are shown in Table 3.

Table 3. Support degrees among experts

\begin{tabular}{|c|c|c|c|c|c|}
\hline Expert & Support Degree & Expert & Support Degree & Expert & Support Degree \\
\hline$h_{1} \rightarrow h_{2}$ & $\frac{2}{15}$ & $h_{1} \rightarrow h_{5}$ & $-\frac{7}{12}$ & $h_{1} \rightarrow h_{7}$ & $\frac{3}{10}$ \\
\hline$h_{2} \rightarrow h_{1}$ & $-\frac{1}{3}$ & $h_{2} \rightarrow h_{4}$ & $\frac{2}{5}$ & $h_{2} \rightarrow h_{5}$ & $\frac{7}{12}$ \\
\hline$h_{2} \rightarrow h_{9}$ & $\frac{1}{6}$ & $h_{3} \rightarrow h_{1}$ & $\frac{3}{10}$ & $h_{3} \rightarrow h_{4}$ & $\frac{1}{6}$ \\
\hline$h_{3} \rightarrow h_{7}$ & $-\frac{1}{3}$ & $h_{3} \rightarrow h_{8}$ & $-\frac{3}{20}$ & $h_{4} \rightarrow h_{5}$ & $\frac{7}{30}$ \\
\hline$h_{5} \rightarrow h_{8}$ & $\frac{1}{15}$ & $h_{6} \rightarrow h_{2}$ & $-\frac{3}{10}$ & $h_{6} \rightarrow h_{4}$ & $-\frac{3}{10}$ \\
\hline$h_{7} \rightarrow h_{4}$ & $-\frac{3}{20}$ & $h_{7} \rightarrow h_{8}$ & $-\frac{7}{30}$ & $h_{8} \rightarrow h_{1}$ & $\frac{1}{6}$ \\
\hline$h_{9} \rightarrow h_{2}$ & $\frac{1}{4}$ & $h_{9} \rightarrow h_{3}$ & $\frac{3}{20}$ & $h_{9} \rightarrow h_{10}$ & $-\frac{1}{5}$ \\
\hline$h_{10} \rightarrow h_{1}$ & $\frac{7}{30}$ & $h_{10} \rightarrow h_{3}$ & $-\frac{1}{5}$ & $h_{10} \rightarrow h_{9}$ & $-\frac{1}{4}$ \\
\hline
\end{tabular}

According to the known support degrees to calculate the $P R$ values by equation (3), after several iterations, the ultimate stability of the $P R$ values as shown in Table 4. Table 4. The PR values of experts

\begin{tabular}{|c|c|c|c|}
\hline Expert & $P R$ & Expert & $P R$ \\
\hline$h_{1}$ & 0.15321 & $h_{2}$ & 0.15044 \\
\hline$h_{3}$ & 0.14947 & $h_{4}$ & 0.15005 \\
\hline$h_{5}$ & 0.15315 & $h_{6}$ & 0.15000 \\
\hline$h_{7}$ & 0.15142 & $h_{8}$ & 0.14913 \\
\hline$h_{9}$ & 0.14894 & $h_{10}$ & 0.14958 \\
\hline
\end{tabular}

From Table 4, the current rank among experts can be seen: $h_{1}>h_{5}>h_{7}>h_{2}>h_{4}>h_{6}>h_{10}>h_{3}>h_{8}>h_{9}$. 


\section{Conclusions}

Real-time and accurately understanding of current viewpoints ranking in order to improve the efficiency of experts in HWME. Therefore, in this paper, viewpoint ranking method on PageRank is proposed, based on information organization model, designing the computational methods of support degrees and PageRank values to rank the experts' viewpoints. The $P R$ value is used to solve the ranking problem of divergent discussion. Finally, examples are used to validate the validity and practicability of the method. The next step will focus on the relevance of experts' comments and discussion topics, in order to prevent the drift of viewpoints and improve the efficiency of discussion.

\section{Acknowledgement}

This work is supported by the National Natural Science Foundation of China (No. 71301081, 61373139, 61572261, 61302157), Natural Science Foundation of Jiangsu Province (No. BK20130877, BK20140895), Postdoctoral Science Foundation of China (No. 2014M551637), Postdoctoral Science Foundation of Jiangsu Province (No. 1401046C).

\section{References}

[1] Xue-sen Qian, Jing-yuan Yu, Ru-wei Dai, A new area of science-Huge open complex system and its methology, Chinese Journal of Nature. 13 (1990) 3-10. (In Chinese)

[2] Xue-sen Qian, Another comment on the open complex giant system, Pattern Recognition and Artificial Intelligence. 4 (1991) 5-8. (In Chinese)

[3] Jing-yuan Yu, Xue-sen Qian, Researches on the open complex giant systems, Systems Engineering-Theory \& Practice. 12 (1992) 8-12. (In Chinese)

[4] Long-bing Cao, Ru-wei Dai, Architecture of Internet-based Hall for Workshop of Meta-synthetic Engineering, Computer Science. 29 (2002) 63-66. (In Chinese)

[5] Jing-yuan Yu, Xiao-ji Zhou, The realization and application of meta-synthesis, Systems Engineering-Theory \& Practice. 22 (2002) 26-32. (In Chinese)

[6] Xiao-hui $\mathrm{Hu}$, The research of methodology and technique for the system implementation of Hall for Workshop of Meta-synthetic Engineering, Systems Engineering-Theory \& Practice. 22 (2002) 1-10. (In Chinese)

[7] Dan-li Wang, Ru-wei Dai, Research on convergence of expert group thought, Journal of Management Sciences in China. 5 (2002) 1-5. (In Chinese)

[8] Xia Chen, Zhi-ping Fan, A new method of group decision ranking based on linguistic judgment matrices, Systems Engineering. 27 (2009) 90-94. (In Chinese)

[9] Min Tan, Yue Shi, Jun-cao Yang, et al, Multi-granularity uncertain linguistic group decision making method based on similarity measure, Computer Science. 43 (2016) 262-265. (In Chinese)

[10] Xiang-qian Feng, Gang Hu, Cui-ping Wei, Consistency analysis of judgment and project ranking in group decision making, Operations Research and Management Science, 16 (2007) 79-82. (In Chinese) 
[11] Karacapilidis N, Pappis C, Computer-supported collaborative argumentation and fuzzy similarity measures in multiple criteria decision making, Computers \& Operations Research. 27 (2000) 653-671.

[12] Gordont F, Prakken H, Walton D, The Carneades model of argument and burden of proof, Artificial Intelligence. 171 (2007) 875-896.

[13] Xing-xue Zhang, Peng-zhu Zhang, Research on visualization of group decision argument opinions distributing--design and development of electronic common brain audiovisual room, Journal of Management Sciences in China. 8 (2005) 15-27. (In Chinese)

[14] Xi-jin Tang, Yi-jun Liu, From group support system to creativity support system, Systems Engineering- Theory \& Practice. 26 (2006) 64-71. (In Chinese)

[15] Ai Wang, Yao-dong Li, Probabilistic mixture model based summarization approach for CWME discussions, Computer Science. 38 (2011) 191-194. (In Chinese)

[16] Jian Zhou, Yao-qin Zhu, Xu-dong Chai, Wei-qing Tang, Approach for analyzing consensus based on cloud model and evidence theory, Systems Engineering-Theory \& Practice. 32 (2012) 2756-2763. (In Chinese)

[17] Zhi-ying Li, Wu Yang, Zhi-jun Xie, Research on PageRank Algorithm, Computer Science. 38 (2011) 185-188. (In Chinese)

[18] Jun-feng Tan, Peng-zhu Zhang, Li-ning Huang, A group argumentation information-structuring model in Hall for Workshop of Meta-synthetic Engineering, Systems Engineering-Theory \& Practice. 25 (2005) 86-99. (In Chinese)

[19] Dong Xiang, Yong Zhao, Yang Chen, Method of structure and fusion for uncertainly seminar information, Computer Science. 39 (2012) 187-190. (In Chinese) 